\title{
Isomérisation du lactose en solution aqueuse et du lactose du lactosérum sur résines échangeuses d'ions
}

\author{
par \\ M. DEMAIMAY et C. BARON* \\ (avec la collaboration technique de Anne ROUSSEAU) \\ Laboratoire de Chimie-Biochimie Alimentaire (E.N.I.T.I.A.A.) \\ Domaine de la Géraudière - 44072 Nantes cedex
}

\section{R és u m é}

L'isomérisation du lactose en lactulose a été effectuée sur une résine échangeuse d'anions du type $\mathrm{AF} 003$ fortement basique, polystyrénique et macroporeuse. L'étude des différents paramètres indique que le débit de la solution de lactose influence peu le taux d'isomérisation. Par contre, ce dernier varie très sensiblement avec la température de la résine d'isomérisation et la concentration en lactose de la solution. Le meilleur pourcentage de lactulose obtenu se situe vers 36 p. 100.

L'application de la méthode au lactosérum nécessite une filtration ou une centrifugation préalable et si le produit n'est pas complètement déminéralisé, l'utilisation de résines de déminéralisation acide et basique. Après décoloration, le produit obtenu, avec 30 p. 100 de lactulose, possède de bonnes propriétés organoleptiques. Une cristallisation fractionnée permet d'enrichir la solution en lactulose.

L'analyse de cet isomérisat de lactosérum effectuée par chromatographie gazeuse permet de mettre en évidence une hydrolyse partielle du lactose et du lactulose, qui conduit à la présence du galactose.

* C. Baron, Laboratoire de Biochimie, E.N.S.B.A.N.A., Campus Universitaire 21000 Dijon. 
On observe de plus l'apparition d'un composé qui a été identifié grâce au couplage chromatographie gazeuse-spectrométrie de masse, comme étant un diholoside, exempt de fructose, mais renfermant dans sa structure soit du galactose pur, soit du glucose pur, soit un mélange des deux.

\section{INTRODUCTION}

Les faibles propriétés fonctionnelles, organoleptiques et techniques du lactose, à savoir : le phénomène d'intolérance $[1,2]$, son faible pouvoir sucrant et sa cristallisation rapide sont des handicaps certains pour la valorisation du lactosérum en alimentation humaine.

Actuellement plusizurs voies sont ouvertes pour la revalorisation de ces produits : la voie microbiologique avec la production de levure et d'autres micro-organismes, et celle de l'hydrolyse enzymatique ou catalytique du lactose, avec la production de sucres liquides.

La mise en évidence par Montgomery [3, 4] et Oosten [5] d'un isomère du lactose : le lactulose ou 4 0- $\beta$-D galactopyranosyl-Dfructose qui apparaît en équilibre avec le lactose en solution basique, ouvre une autre voie. En effet, le lactulose est un édulcorant non calorifique, car il résiste aux enzymes hydrolysantes. Il possède un pouvoir sucrant proche du glucose. Il est très soluble dans l'eau et difficilement cristallisable. En outre, il possède en solution une bonne viscosité. Il est enfin intéressant de noter qu'il intervient au niveau de la flore bifidogène de l'intestin et empêche la formation de substances toxiques. Ces qualités en font un produit alimentaire intéressant [6]. De plus, comme l'indique Baglioni [7], l'ingestion de plusieurs fois la dose humaine supposée maximale ne laisse apparaître aucun effet toxique.

La préparation conventionnelle du lactulose consiste à ajouter un agent alcalin tel que $\mathrm{Ca}(\mathrm{OH})_{2}, \mathrm{KOH}, \mathrm{K}_{2} \mathrm{HPO}_{4}, \mathrm{Ba}(\mathrm{OH})_{2}, 8 \mathrm{H}_{2} \mathrm{O}$, etc., à une solution de lactose dans des proportions de 2 à 5 p. 100. La solution est chauffée et le temps nécessaire à l'équilibre des deux isomères varie de $10 \mathrm{mn}$ à $2 \mathrm{j}$ en fonction de la température : $30^{\circ} \mathrm{C}$ à $130^{\circ} \mathrm{C}$ [8].

Récemment, plusieurs procédés concernant la production de lactulose à partir du lactose pur ont été décrits. On peut citer : l'utilisation des sulfites [9], des aluminates de sodium et calcium [10], des tétraborates de sodium [11] et de l'hydroxyde de sodium [12].

Compte tenu du procédé proposé par Sato et al. [6], dans lequel il est fait mention de l'utilisation des résines échangeuses d'anions comme catalyseur pour l'isomérisation du lactose en lactulose, et 
des travaux effectués par Petersen [13] sur l'isomérisation du glucose en fructose à l'aide des résines anioniques, nous avons étudié ici l'influence des différents paramètres (type de résines, concentration en lactose, température et débit) qui sont susceptibles d'intervenir sur le taux d'isomérisation du lactose lorsqu'on utilise une résine anionique comme catalyseur.

D'autre part, la mise au point de ce procédé est développée au niveau du lactosérum et les conditions d'obtention d'un produit possédant de bonnes caractéristiques alimentaires sont définies.

La composition de cet isomérisat de lactosérum est enfin étudiée.

\section{MATERIEL ET METHODES}

\section{A. Matières premières}

- Lactose hydraté $\mathrm{C}_{12} \mathrm{H}_{22} \mathrm{O}_{11}, \mathrm{H}_{2} \mathrm{O}$ Merck.

- Lactosérum en poudre déprotéiné. Ce produit est obtenu par ultrafiltration du lactosérum liquide puis évaporation sous vide (méthode Spray), Coopérative de la Prospérité Fermière, Arras.

$$
\begin{aligned}
& \text { Caractéristiques du produit : humidité : } \quad 3 \text { p. } 100 \\
& \text { cendres : } \quad 10 \text { p. } 100 \\
& \text { matières azotées : } 5 \text { p. } 100 \\
& \text { lactose : } \quad 82 \text { p. } 100
\end{aligned}
$$

- Lactosérum liquide déprotéiné et déminéralisé à 65 p. 100 , Coopérative de la Prospérité Fermière.

$$
\begin{aligned}
& \text { Caractéristiques du produit : humidité : } \quad 3 \text { p. } 100 \\
& \text { cendres: } \quad 2 \text { p. } 100 \\
& \text { matières azotées : } 6 \text { p. } 100 \\
& \text { lactose : } \quad 88 \text { p. } 100
\end{aligned}
$$

- Résines échangeuses d'ions, type anionique : AF 003 et AM 006, type cationique C 005, Société Applexion, 9, avenue de la Mauldre 78680 Epone.

\section{B. Préparation des échantillons}

- Solution no 1 : solution aqueuse à $250 \mathrm{~g} / 1$ de lactose et $20 \mathrm{~g} / 1$ de méso-inositol (témoin interne).

- Solution no 2 : solution de lactosérum en poudre à $250 \mathrm{~g} / \mathrm{l}$ et $20 \mathrm{~g} / 1$ de méso-inositol.

- Solution no 3 : lactosérum liquide concentré à $18 \mathrm{p} .100$ de matière sèche $(157 \mathrm{~g} / 1$ de lactose $)$. 


\section{Protocole d'isomérisation}

La mise au point de la méthode et l'étude des différents paramètres qui interviennent sur le taux d'isomérisation ont été effectuées sur une colonne en verre à double paroi à l'aide de la solution de lactose $\mathrm{n}^{\circ} 1$.

Cette solution est préchauffée avant son passage sur la résine, qui est elle-même calorifugée à l'aide d'un cryostat Haake FK2, contenant de l'éthylène glycol.

On règle le débit à l'aide d'une pompe péristaltique.

En sortie de colonne, la solution est recueillie par fractions de $9 \mathrm{ml}$ à l'aide d'un collecteur.

Deux types de résines ont été utilisées pour catalyser la réaction d'isomérisation :

- AF 003 : anionique, fortement basique, polystyrénique et macroporeuse ;

- AM 006 : anionique, moyennement basique, polystyrénique.

L'isomérisation du lactosérum nécessite une opération préalable de déminéralisation totale qui est effectuée à l'aide d'une résine cationique forte C 005 et d'une résine anionique AM 006.

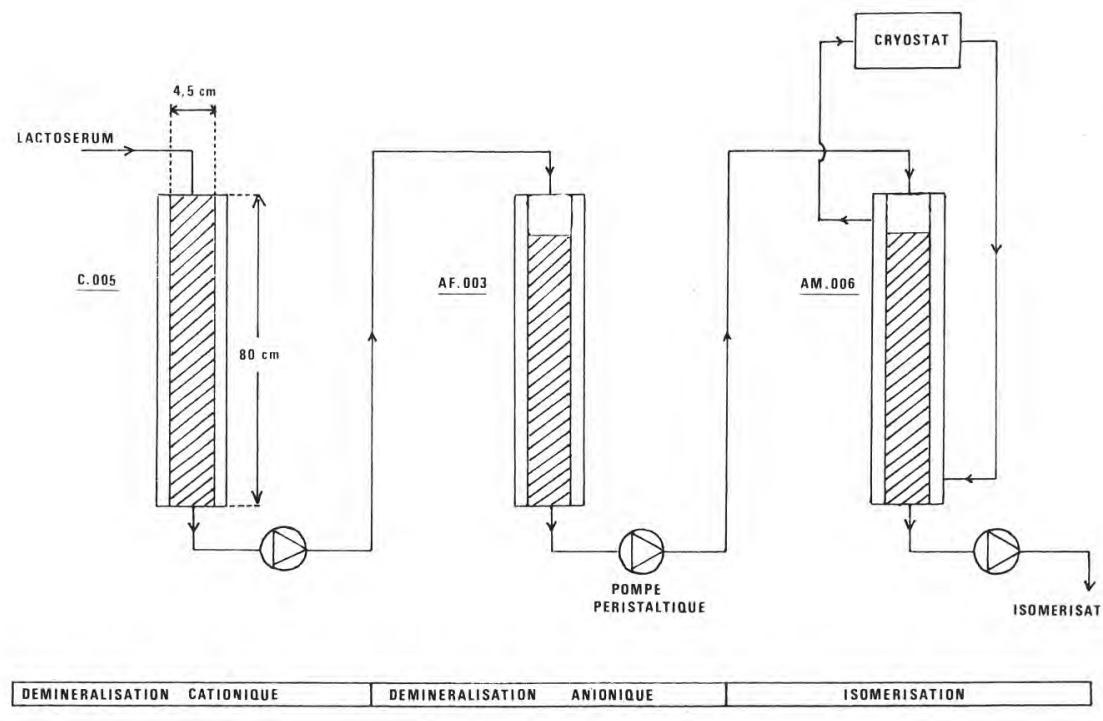

fig. 1

Schéma de l'appareillage permettant la déminéralisation complète du lactosérum et l'isomérisation du lactose. 
Le volume de la résine C 005 est plus important que les autres, car en plus de sa fonction de déminéralisation cationique, elle retient les acides aminés libres du lactosérum (cette dernière opération évite les interactions sucres-acides aminés qui sont responsables de réactions du type Maillard).

L'appareillage est schématisé sur la figure 1.

D. Techniques d'analyse des sucres par chromatographie en phase gazeuse

La chromatographie en phase gazeuse (C.P.G.) permet de doser qualitativement et quantitativement en une seule analyse les différents sucres présents dans l'isomérisat. Elle est donc préférable à la plupart des autres méthodes enzymatiques et colorimétriques qui ne permettent pas un dosage simultané des différents sucres : galactose, lactulose, lactose, etc.

Le chromatographe utilisé est un appareil Packard, model 419, équipé d'un détecteur à ionisation de flamme. L'intensité du détecteur est de $2,5 \cdot 10^{-10} \mathrm{~A}$. L'injection des échantillons se fait directement en tête de colonne à l'aide d'une seringue S.G.E. de $5 \mu$ l. La colonne spiralée en verre Pyrex a $3 \mathrm{~m}$ de long et $3 \mathrm{~mm}$ de diamètre interne, elle contient de l'OV-17 à 5 p. 100. Support : Supelcoport 100-120 mesh.

La température de l'injecteur et du détecteur est de $250^{\circ} \mathrm{C}$.

Débits : gaz vecteur (azote) : $30 \mathrm{ml} / \mathrm{mn}$;

hydrogène : $30 \mathrm{ml} / \mathrm{mn}$;

air : $300 \mathrm{ml} / \mathrm{mn}$.

En nous appuyant sur la méthode de Zürcher [14, 15], nous avons mis au point le protocole opératoire suivant :

- prélever $5 \mu \mathrm{l}$ du produit à analyser ;

- évaporer à sec à $80^{\circ} \mathrm{C}$;

- ajouter $50 \mu \mathrm{l}$ de pyridine et $100 \mu \mathrm{l}$ de N,O-bis-(triméthylsilyl)trifluoro-acétamide (B.S.T.F.A.) à 1 p. 100 de triméthylchlorosylane (T.M.C.S.) ;

- porter à l'étuve $4 \mathrm{~h}$ à $80^{\circ} \mathrm{C}$;

- conserver le dérivé silylé 3 semaines maximum à $4^{\circ} \mathrm{C}$.

Les conditions d'analyse sont les suivantes :

- température du four : $180^{\circ} \mathrm{C}$ pendant $10 \mathrm{mn}$ puis programmation de $5^{\circ} \mathrm{C} / \mathrm{mn}$ jusqu'à $230^{\circ} \mathrm{C}$;

- injection de 1 à $2 \mu 1$ de l'échantillon ;

- la durée d'une analyse est de $40 \mathrm{mn}$.

Les analyses en couplage chromatographie gazeuse - spectrométrie de masse ont été effectuées sur un spectromètre Varian Mat 311. La colonne spiralée en métal a $1,50 \mathrm{~m}$ de long sur $2 \mathrm{~mm}$ de diamètre 
interne et elle contient de l'OV-17 à 2 p. 100 sur support Chromosorb Q 80/100 mesh. Le voltage d'accélération est de $70 \mathrm{eV}$, la température de la source d'ions de $190^{\circ} \mathrm{C}$.

\section{RESULTATS ET DISCUSSION}

\section{A. Essais sur le lactose}

Dans un premier temps, nous avons observé qu'une résine échangeuse d'anions moyennement basique avait un effet catalytique nettement inférieur à celui d'une résine échangeuse d'anions fortement basique.

Ainsi à $60^{\circ} \mathrm{C}$, la résine fortement basique du type $\mathrm{AF} 003$ a un rendement d'isomérisation de 24 p. 100, deux fois plus élevé qu'une résine moyennement basique du type AM 006.

Dans un second temps, nous avons déterminé l'influence des facteurs température, débit et concentration en lactose sur le taux d'isomérisation.

La résine utilisée est du type AF 003.

Température : les essais ont été menés à l'aide de la solution $\mathrm{n}^{\circ} 1$, le débit est fixé à $400 \mathrm{ml} / \mathrm{h}$.

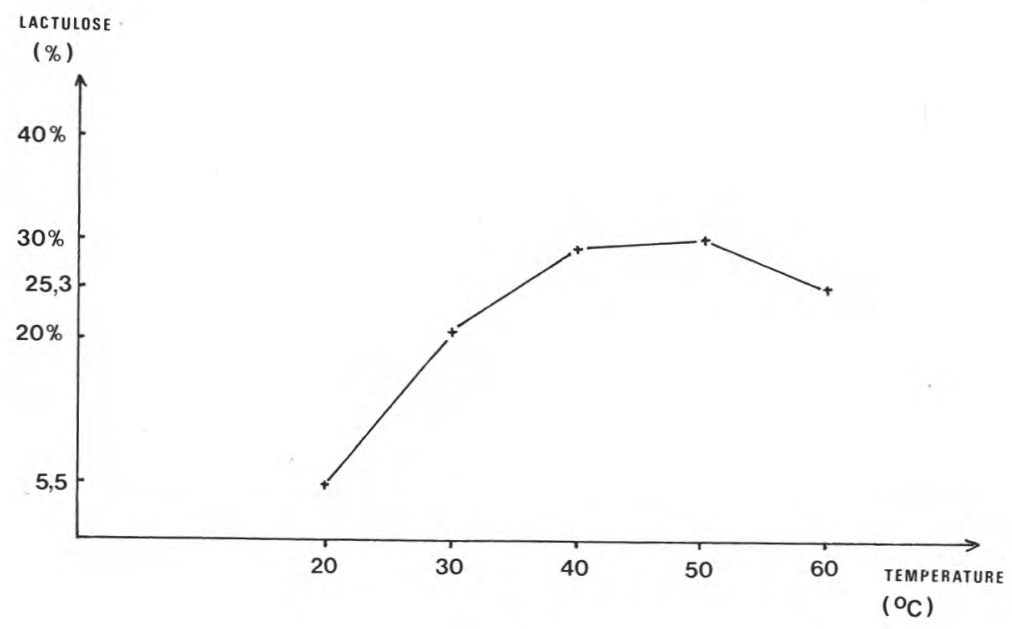

fig. 2

Courbe représentant les variations du taux d'isomérisation du lactose en lactulose en fonction de la température. 
La figure 2 permet de constater que le taux d'isomérisation est maximum pour une température de $50^{\circ} \mathrm{C}$. Cependant, à cette température, on observe une coloration en jaune pâle du produit en sortie de colonne. Il apparaît donc préférable de fixer la température à $40^{\circ} \mathrm{C}$. Dans ces conditions, le taux d'isomérisation reste satisfaisant et le produit incolore.

Débit : les essais sont menés avec la solution n 1 à $40^{\circ} \mathrm{C}$.

La figure 3 montre que le taux d'isomérisation varie peu en fonction du débit. Il est maximum à $200 \mathrm{ml} / \mathrm{h}$ et égal à 27,8 p. 100 . Cela semble s'expliquer par le fait que l'on n'observe pas de variation sensible du $\mathrm{pH}$ au cours de l'expérience. La résine conserve alors tout son pouvoir basique d'isomérisation quel que soit le débit de la solution de lactose.

Compte tenu de la faible variation observée, le débit a été maintenu à $400 \mathrm{ml} / \mathrm{h}$ pour les autres expériences.

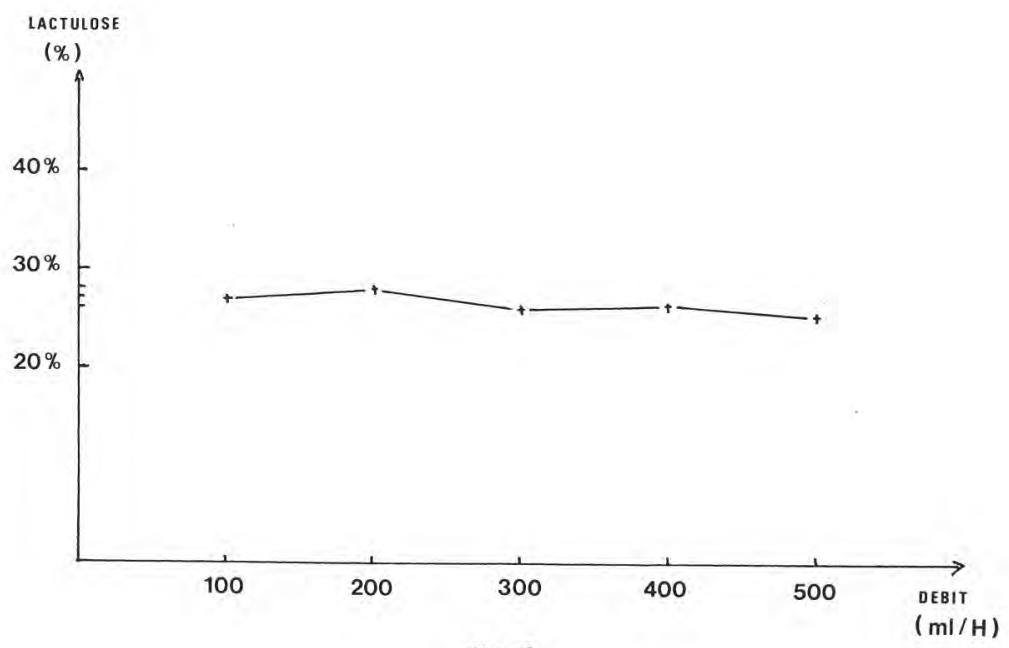

fig. 3

Courbe représentant les variations du taux d'isomérisation du lactose en lactulose en fonction du débit.

Concentration en lactose : les essais sont menés à $40^{\circ} \mathrm{C}$.

Sur la figure 4 , on constate une nette influence de la concentration en lactose sur le taux d'isomérisation. Les faibles concentrations conduisent aux meilleurs pourcentages d'isomérisation avec un maximum de $36 \mathrm{p} .100$ pour la solution à $100 \mathrm{~g} / 1$. Cependant, aux très faibles concentrations et en particulier à $50 \mathrm{~g} / \mathrm{l}$, il apparaît un phénomène de rétention total des diholosides dans la résine. 
Les meilleures conditions d'isomérisation du lactose sont donc obtenues en fixant la température à $40^{\circ} \mathrm{C}$, le débit entre 200 et $400 \mathrm{ml} / \mathrm{h}$ et la concentration en lactose vers $100 \mathrm{~g} / \mathrm{l}$. Le taux moyen d'isomérisation est alors de 36 p. 100.

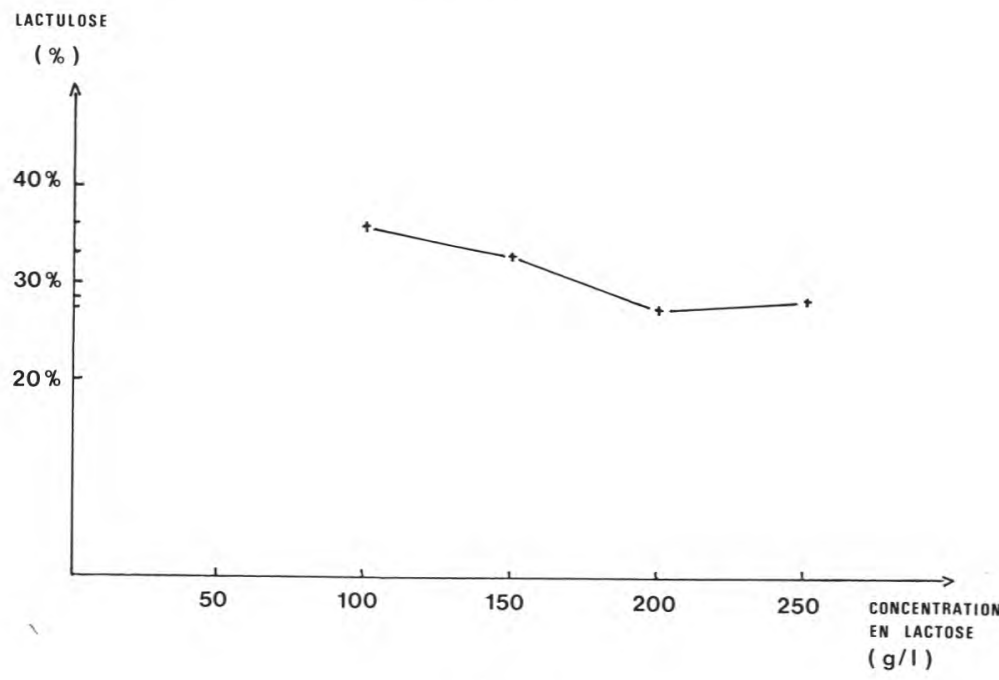

fig. 4

Courbe représentant les variations du taux d'isomérisation du lactose en lactulose en fonction de la concentration en lactose.

\section{B. Essais sur le lactosérum}

Le rendement industriel du système est favorisé en utilisant des concentrations relativement élevées en lactosérum. De ce fait, c'est avec les solutions $n^{\text {os }} 2$ et 3 que l'influence de la température sur le taux d'isomérisation a été vérifiée. Les essais ont été menés dans les conditions suivantes : le volume de lactosérum traité correspondait à une fois et demie le volume de résine des trois colonnes. On travaillait ainsi dans des conditions proches de celles d'un passage en continu. Le débit était fixé à $400 \mathrm{ml} / \mathrm{h}$.

Avec la solution $\mathrm{n}^{\circ} 2$, le pourcentage de lactulose obtenu est de 32 p. 100 à $40^{\circ} \mathrm{C}$ et de 24 p. 100 à $60^{\circ} \mathrm{C}$.

Ces résultats confirment les expériences effectuées sur la solution $\mathrm{n}^{\circ} 1$, à savoir un taux d'isomérisation sensiblement plus élevé à $40^{\circ} \mathrm{C}$ qu'à $60^{\circ} \mathrm{C}$. Une étude analogue avec la solution $\mathrm{n}^{\circ} 3$ aboutit aux mêmes conclusions. A $40^{\circ} \mathrm{C}$, le pourcentage de lactulose est de 29 p. 100 et à $60^{\circ} \mathrm{C}$ de 21 p. 100. 
L'ensemble des échantillons de lactosérum ainsi traités conduit donc à un pourcentage moyen d'isomérisation de 30 p. 100, la température étant maintenue à $40^{\circ} \mathrm{C}$ et le débit à $400 \mathrm{ml} / \mathrm{h}$.

Il est intéressant de noter que la technique d'analyse des sucres sous forme de dérivés volatils permet de mettre en évidence, comme on le remarque sur la figure 5, des pics chromatographiques ne correspondant ni au lactose, ni au lactulose.

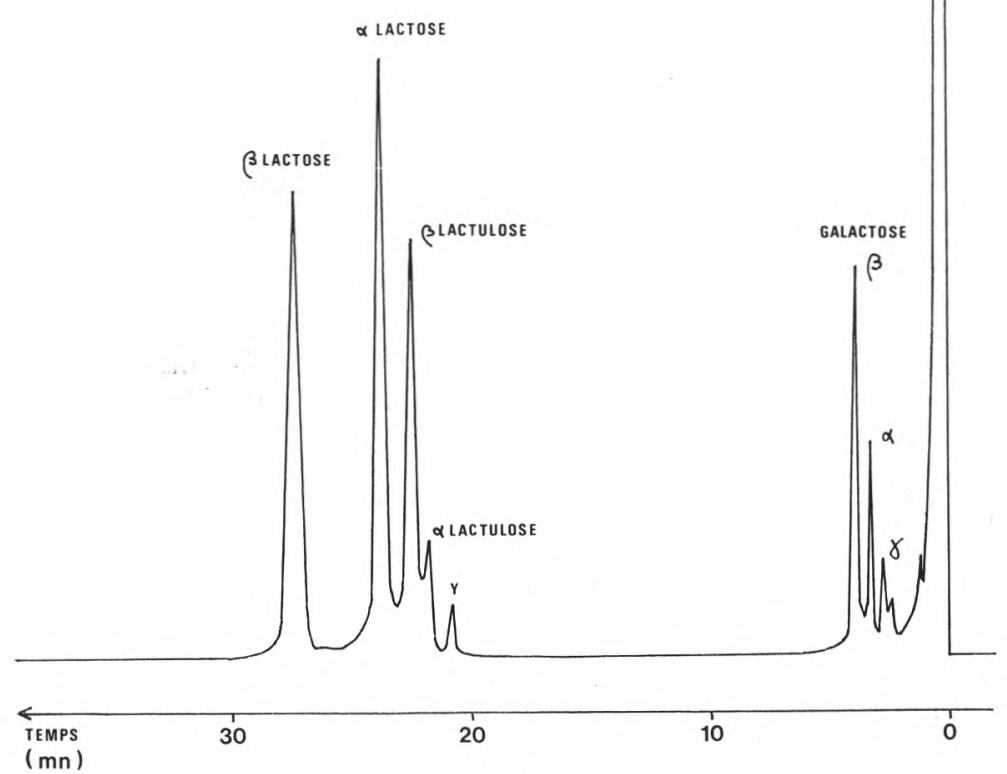

fig. 5

Chromatogramme représentant les différents sucres sous forme de dérivés silylés après passage de la solution de lactosérum liquide sur les trois colonnes de résines. La température de la résine d'isomérisation est fixée à $40^{\circ} \mathrm{C}$. Le débit de la solution est de $400 \mathrm{ml} / \mathrm{h}$. 
Les trois premiers ont été identifiés grâce à leurs temps de rétention et par des essais de surcharge, comme étant les isomères $\alpha$, $\beta$ et $\gamma$ du galactose.

L'étude du spectre de masse du composé $\mathrm{Y}$, obtenu grâce au couplage chromatographie gazeuse-spectrométrie de masse, nous permet d'affirmer qu'il s'agit d'un diholoside. L'identification de sa structure est en cours, mais la présence de fructose dans ce diholoside étant exclue, les seules possibilités d'arrangement semblent du type : galactose + galactose, glucose + glucose et glucose + galactose

Pendant le passage des solutions $n^{\text {os }} 2$ et 3 de lactosérum sur la résine de déminéralisation anionique (AM 006), un trouble apparaît au niveau des grains de résine. Ce trouble persiste jusqu'à la sortie de la colonne d'isomérisation et donne au produit final un aspect qui nuit à sa présentation. L'origine du phénomène pourrait être attribuée à la faible quantité de protéines restant dans le produit, qui n'est pas retenue sur la résine cationique $(\mathrm{C}$ 005) et qui précipite par effet isoélectrique lors du mélange de la solution acide et basique. La présence de polyphosphates pourrait en être également la cause.

Il est possible de supprimer le trouble en agissant directement sur le lactosérum avant la déminéralisation. Trois techniques ont été envisagées. La première était une ultrafiltration, abandonnée à cause de sa lenteur, la seconde une centrifugation à $22000 \mathrm{~g}$ pendant $10 \mathrm{mn}$, et la troisième une filtration sur filtre stérile. Le lactosérum après centrifugation, ou filtration stérile, déminéralisation et isomérisation conduit à un produit parfaitement limpide.

D'autre part, sur la colonne d'isomérisation, on constate que le produit se colore en jaune pâle, couleur qui semble provenir d'un début de dégradation des sucres en milieu basique et chaud.

La décoloration de l'isomérisat se fait sans difficulté, par passage sur une résine anionique faiblement basique du type AM 006.

\section{Concentration, conservation et cristallisation sélective du lactose dans les isomérisats de lactosérum}

Les isomérisats de lactosérum après décoloration des solutions $n^{\text {os }} 2$ et 3 sont concentrés à 70 p. 100 de matières sèches à l'aide d'un évaporateur rotatif sous vide. Les produits obtenus sont limpides, incolores et de saveur sucrée.

On remarque toutefois que le lactose contenu dans l'isomérisat issu de la solution $n^{\circ} 2$ (lactosérum en poudre), cristallise dès la concentration, alors que celui de la solution $n^{\circ} 3$ (lactosérum liquide) se conserve à l'état liquide pendant plusieurs jours. 
La précristallisation du lactose dans la poudre de lactosérum semble donc favoriser sa recristallisation par la suite.

Ce phénomène de cristallisation rapide et sélective du lactose par concentration sous vide permet d'obtenir si nécessaire un isomérisat très enrichi en lactulose.

Le phénomène est spontané lorsqu'il s'agit d'un isomérisat de lactose pur ou de lactosérum en poudre. Si l'on désire une cristallisation immédiate du lactose des isomérisats de lactosérum liquide, il est nécessaire d'effectuer une amorce avec des cristaux de lactose pur.

\section{Remerciements}

Les auteurs remercient la Coopérative de La Prospérité Fermière et la Société Applexion pour les produits et les échantillons qu'ils ont bien voulu mettre à leurs dispositions.

\section{S u m m a r y}

The isomerisation of lactose into lactulose has been carried out using an anion exchange resin (AF 003 type) highly basic, polystyrenic and macroporous.

The study of the various parameters shows that the flow of the lactose little influence on the isomerisation rate. But this rate varies apprecially with the temperature of the isomerisation resin and the lactose concentration of the solution. The best percentage of lactulose obtained is around 36 p. 100.

The application of the method to whey requires a filtration or a previous centrifugation, an if the product is not completly demineralized the use of acid and basic demineralization resins. After decolorization, the product thus obtained, with 30 p. 100 of lactulose, has good organoleptic properties.

Crystallization and removal of lactose favours the lactulose enrichment of the solution.

The analysis of this whey isomerisate carried out by gas chromatography shows a partial hydrolyse of lactose and lactulose revealing galactose.

Moreover, there can be noticed a compound identified by means of gas chromatography and mass spectrometry as being a dissacharide without fructose, but whose structure contains either pure galactose, or pure glucose, or still a mixture of both. 


\section{Bibliographie}

[1] 1971. - Lactose intolerance and milk drinking. Habits gastroenterology, $60,4$.

[2] 1972. - Lactose tolerance in a slavic population. The Amer. J. of digestive disensed, New series, 17, 1.

[3] Montgomery, Hudson (1930). - J. Am. Chem. Soc., 52, 2101.

[4] Montgomery (E. D.) (1962). - Rearrangement of lactose to lactulose in alkaline solution. Methods in carbohydrate chemistry. Whistler and Wolfrom Editors, Academic Press, 325-328.

[5] Oosten (1967). - Rec. Trav. Chim., Pays-Bas, 86, 673.

[6] Sato (Y.), Miyake (T.), Sakai (S.), Mitsuhashi (M.) (1970). - Procédé pour la production de lactose. Brevet $\mathrm{n}^{\circ} 2053323$.

[7] Baglioni (A.), Dubini (F.) (1976). - Lattulosio : valutazione tossicologica. Boll. Chim. Farm, 115, 596-606.

[8] Johnson (J.) (1976). - Lactulose; specialized sugars for the food industry. Ed. Noyes Data Corporation, Park Ridges, 264-277.

[9] Nitsch (E.), Muhlвock (S.) (1972). - Epimerization of lactose with sulfites. U.S. Patent $n^{\circ} 3707534$.

[10] Guth (J. H.), Tumerman (L.) (1970). - Isomerisation of lactose with aluminates. U.S. Patent $n^{\circ} 3546206$.

[11] Carubelli (R.). (1970). - Isomerisation of lactose with Alcali metal tetraborates. U.S. Patent $n^{\circ} 3505309$.

[12] Nagasawa (T.), Tomita (M.), Tamura (Y.), Obayashi (T.), Mizota (T.) (1974). Isomerisation of lactose with sodium hydroxyde as catalyst. U.S. Patent $n^{\circ} 3816174$.

[13] Petersen (1975). - Isomerisation with anion exchange resins. Edible starches and Starch-derived syrups. Ed. Noyes Data Corporation, Park Ridges, 366-369.

[14] ZuRcher (K.), HADORN (H.) (1974). - Versuche zur Herstellung und gaschromatographischen Trennung der Zucker-Silyläther. Deutsche LebensmittelRundschau, 70, Jahrg, Heft 12.

[15] ZuRcheR (K.), HAdoRN (H.) (1975). - Optimisierung der Versuchsbedingungen zur silylierung der Zucker mit BSA und HDMS und der gaschromatographischen Trennung. Deutsche Lebensmittel-Rundschau, 71, Jahrg, Heft 2. 